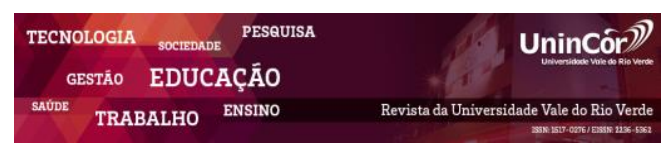

Revista da Universidade Vale do Rio Verde ISSN: 1517-0276 / EISSN: 2236-5362 Vol. 16 | n. 1 | Ano 2018

Fernanda Breda Universidade de Passo Fundo - UPF fernandafbreda@gmail.com

Elizabete Casagrande Lazarotto Universidade de Passo Fundo - UPF e.lazarotto@hotmail.com

Cassiana Maris Lima Cruz Universidade de Passo Fundo - UPF cassiana@upf.br

Ana Claudia Machado Padilha Universidade de Passo Fundo - UPF anapadilha@upf.br

Lidiane Zambenedetti Universidade de P asso Fundo - UPF lidiane.zambenedetti@erechim.ifrs.edu.br

\section{REFLEXÕES ACERCA DA COMUNICAÇÃO ORGANIZACIONAL COMO FERRAMENTA ESTRATÉGICA EM REDES DE COOPERAÇÃO}

\section{RESUMO}

O assunto sobre redes de cooperação tem se tornado cada vez mais abordado nos estudos organizacionais em busca de maior vantagem competitiva. $\mathrm{O}$ mesmo ocorre com a abordagem da comunicação como ferramenta de gestão nas organizações. Neste sentido, o presente ensaio teórico tem como objetivo estudar a relação existente entre comunicação organizacional, estratégias de redes e redes de cooperação. Para tal, desenvolveu-se uma pesquisa bibliográfica abordando cada um desses tópicos. Com base na pesquisa realizada, evidenciou-se que os temas estão diretamente relacionados, uma vez que, é por meio da comunicação organizacional que ocorre a interação entre os participantes de uma rede de cooperação, bem como a disseminação dos objetivos em comum. A temática abordada mostra-se relevante devido a escassez de estudos que relacionem a comunicação organizacional com as redes de cooperação, bem como a comunicação como ferramenta estratégica para formação de redes.

Palavras-chave: Comunicação Organizacional. Estratégia de redes. Redes de cooperação. Vantagem competitiva. Comunicação em redes de cooperação.

\section{REFLECTIONS ON ORGANIZATIONAL COMMUNICATION AS A STRATEGIC TOOL IN COOPERATION NETWORKS}

\begin{abstract}
The subject of cooperation networks has become increasingly addressed in organizational studies seeking greater competitive advantage. The same applies to the communication approach as a management tool in organizations. In this sense, this theoretical essay aims to study the relationship between organizational communication, networking strategies and cooperation networks. To this end, a literature was developed addressing each of these topics. Based on research conducted, it became clear that the issues are directly related, since it is through the organizational communication is the interaction between the participants of a network of cooperation and the spread of common goals. The theme addressed shows to be relevant due to lack of studies that relate to organizational communication with the networks of cooperation and communication as a strategic tool for networking.
\end{abstract}

Keywords: Organizational communication. Networking strategy. Cooperation networks. Competitive advantage. Communication in cooperation networks. 


\section{INTRODUÇÃO}

A comunicação tem se tornado um tema cada vez mais relevante nos estudos organizacionais, face as grandes transformações tecnológicas, busca de vantagem competitiva, tecnologia da informação e aumento da produtividade nas organizações (SCROFERNEKER, 2000).

A comunicação, inserida nas principais funções administrativas, de planejamento, organização, direção e controle e nas relações de organizações, constitui a alma da organização, visto que promove relações de entendimento, permitindo a interação entre todos os recursos humanos, materiais e financeiros em busca dos objetivos previamente estabelecidos (WELS, 2005; CORREA, 2010).

Segundo Gonçalves (2005), a comunicação torna-se um instrumento indispensável de gestão, permitindo que a organização realize seus projetos da melhor forma. O processo de comunicação, quando se trata de rede de cooperação empresarial, é ainda mais complexo quando comparado com organizações que não cooperam, pois relaciona diversas empresas e estas passam a constituir uma nova organização (CORRÊA, 2010). Nessa perspectiva, o desafio de compreender a posição estratégica da comunicação nos estudos organizacionais (KUNSCH, 2006), torna-se evidente, pois articular interesses individuais agora em interesse comuns requer gestão com foco em processos comunicacionais que enfatizem os princípios e a estratégia organizacional.

O processo de formação de redes de cooperação é, ainda, uma alternativa para atender aos desafios impostos pelo mercado (EBERS, 1997) e pela nova economia, cuja característica principal é a transição da eficiência individual para a eficiência coletiva, onde a competitividade está cada vez mais relacionada ao desempenho de redes interorganizacionais em relação às empresas isoladas (FLEURY; FLEURY, 2003). As organizações buscam, em relações de parcerias ou cooperação, melhorar sua capacidade de interdependência a fim de complementar suas potencialidades e atingir a eficácia organizacional, aumentando, desta forma, sua competitividade (PEREIRA; PEDROZO, 2005).

Apesar do crescente interesse pelo tema de redes de cooperação na sociedade contemporânea, o fenômeno social das redes não é recente, visto que as inter-relações entre indivíduos e organizações sempre existiram, a partir da existência da humanidade (BALESTRIN; VARGAS, 2003). Entretanto, a estratégia voltada aos modelos competitivos acabou restringindo o estudo das organizações de forma individual. Para os autores, por meio da configuração em rede, as empresas tem uma forma eficaz para alcançarem competitividade nos mercados através de um complexo ordenamento de relacionamentos no qual as firmas estabelecem inter-relações. Estas práticas necessitam de comunicação, dessa forma, as 
redes de cooperação estão intimamente ligadas à comunicação.

Neste sentido, o presente ensaio teórico tem como objetivo estudar a relação existente entre comunicação organizacional, estratégias de redes e redes de cooperação por meio de abordagens conceituais e teóricas. A relevância do estudo está pautada nas poucas evidências de pesquisas que relacionem comunicação organizacional aplicada em redes de cooperação empresarial (CORRÊA, 2010).

Este ensaio teórico está estruturado em seis partes, além desta introdução. Sendo que, primeiramente é apresentado o método que caracteriza o estudo, seguido das abordagens de comunicação organizacional, estratégias para formação de redes de cooperação, redes de cooperação. Posteriormente apresenta-se uma conexão entre os assuntos, comunicação organizacional em redes de cooperação e, por fim, apresentam-se as considerações finais.

\section{MÉTODO}

De forma a alcançar o objetivo proposto neste ensaio teórico foi realizada uma pesquisa bibliográfica que se caracteriza como fonte secundária de coleta de dados (GIL, 2002). Para tanto, recorreu-se as premissas da narrativa, que não utiliza critérios explícitos e sistemáticos para a busca e análise crítica da literatura (Cook, Mulrow \& Raynes, 1997). Assim, foi feita a partir do levantamento de abordagens conceituais e teóricas utilizando as palavras chaves "comunicação" e "rede de cooperação", "comunicação" e "redes interorganizacionais", publicados em periódicos internacionais e nacionais, livros, dissertações de mestrado e teses de doutorado, conforme orientam Cook, Mulrow \& Raynes (1997).

Os artigos nacionais foram selecionados a partir de eventos da área de Administração como os vinculados a Associação Nacional de Pós-Graduação e Pesquisa em Administração (Anpad) e da área da Comunicação Social como o Congresso Brasileiro Científico de Comunicação Organizacional e de Relações Públicas - Abrapcorp. A pesquisa internacional foi realizada nas bases de dados: Emerald, Science Direct e Scopus, pertencendo os artigos selecionados a revistas indexadas.

O estudo também caracterizou-se como exploratório, uma vez que proporcionou maior familiaridade com uma temática pouco discutida (CRESWELL, 2007).

\section{COMUNICAÇÃO ORGANIZACIONAL}

Na tentativa de analisar os fenômenos e processos que acontecem nas organizações, a tradicional visão de gestão, de natureza predominantemente funcionalista, privilegia a crença no poder da estrutura ao invés da compreensão do papel da agência humana na construção da realidade organizacional (DA SILVA; OLIVEIRA, 2008). Assim, construir uma linguagem a fim de lidar com a complexidade de fenômenos organizacionais e de gestão, levando em conta as contribuições e conhecimentos oriundos de outras áreas do conhecimento científico, torna-se um caminho que a ciência da Administração tem como 
desafio na contemporaneidade. Essa dinâmica de conceitos, especialmente no que se refere à comunicação organizacional como ferramenta de estratégia, torna as questões de análise conceitual ainda mais complexas, em virtude da escassez de estudos sobre o tema no campo da Administração, conforme revela Corrêa (2010) em sua pesquisa.

É importante conceituar comunicação organizacional em virtude da importância que esta vem assumindo face às grandes transformações contemporâneas (processos de globalização, revolução tecnológica e de tecnologias de informação, aumento da produtividade e competitividade) e, sobretudo, para demonstrar e evidenciar sua contribuição nas organizações (SCROFERNEKER, 2000).

Para que isto seja possível, é necessário tomar a comunicação como uma variável, sendo um fator que acontece dentro da estrutura da organização (KUNSCH, 2006).

O Quadro 1 apresenta uma síntese dos conceitos de Comunicação Organizacional na perspectivas dos diversos autores.

Quadro 1 - Síntese dos conceitos de comunicação organizacional

\begin{tabular}{|c|l|}
\hline Autor & \multicolumn{1}{|c|}{$\begin{array}{c}\text { Conceito de Comunicação } \\
\text { Organizacional }\end{array}$} \\
\hline Kreps & $\begin{array}{l}\text { Comunicação organizacional é o processo } \\
\text { onde os membros de determinada } \\
\text { organização obtêm informações sobre a } \\
\text { mesma e as mudanças que nela ocorrem. }\end{array}$ \\
\hline Riel & $\begin{array}{l}\text { Comunicação organizacional envolve } \\
\text { todos os processos de relações públicas, } \\
\text { estratégias organizacionais, marketing, } \\
\text { propaganda e comunicação interna e } \\
\text { externa. É caracterizado especialmente } \\
\text { por um público com o qual a organização } \\
\text { se relaciona e depende. }\end{array}$ \\
Daniels; & $\begin{array}{l}\text { Modelo tradicional (processo } \\
\text { comunicacional e à eficiência } \\
\text { organizacional; } \\
\text { interpretativo(organizações } \\
\text { culturas) e crítico (organização como } \\
\text { instrumento de opressão). }\end{array}$ \\
\hline
\end{tabular}

\begin{tabular}{|c|l|}
\hline & \begin{tabular}{l} 
Comunicação organizacional como: (i) \\
transferência de informação; (ii) processo \\
transacional, com foco central no \\
Eisenberg; \\
feedback; (iii) estratégia de controle, onde \\
o comunicador competente é aquele que \\
Jr. (1997) \\
determina as estratégias para a realização \\
de múltiplos objetivos na organização; \\
(iv) equilíbrio entre criatividade e \\
constrangimento/coação/sujeição \\
(constraint) e; (v) espaço de diálogo. \\
\hline Bueno \\
$\begin{array}{l}\text { Conjunto complexo de atividades, ações, } \\
\text { estratégias, produtos e processos } \\
\text { desenvolvidos para reforçar a imagem de } \\
\text { uma empresa ou entidade junto aos seus } \\
\text { públicos de interesse ou opinião pública. }\end{array}$ \\
\hline Comunicar é um processo inerente às \\
organizações e às empresas, que abrange \\
todas as formas de comunicação para \\
relacionar-se e interagir com seus \\
públicos, desde os aspectos internos, \\
considerando as relações interpessoais, \\
estilos de gestão, processos de hierarquia; \\
até os externos, destacando as relações de \\
competitividade entre setores, ações e \\
estratégias de marketing, posicionamento \\
de consumidor e mercado.
\end{tabular} \\
\hline Kunsch \\
(2006)
\end{tabular}

Fonte: Elaborado pelas autoras (2016).

Os processos comunicativos, na visão dos autores, são evidentes, entretanto, eles não devem ser compreendidos como complementos da estratégia organizacional, mas sim, como componentes essenciais na construção e no delineamento das estratégias da empresa.

\section{A FORMAÇÃO DA ESTRATÉGIA DE REDES}

O estudo sobre a formação da estratégia organizacional é essencial para o entendimento das estratégias que contribuem para que as organizações cooperem e formem redes em busca de maior competitividade, complementariedades de recursos e atinjam objetivos em comum.

Porter (1986), fundamentado no modelo estruturalista da indústria predominante na época, destaca que a escolha da estratégia 
depende de uma análise sistemática do ambiente concorrencial em um determinado segmento. Esta análise é pautada no modelo das cinco forças competitivas definidos pelo autor: a) ameaça de novos entrantes; b) poder de barganha dos fornecedores; c) poder de barganha dos compradores; d) ameaça de produtos substitutos e; e) nível de rivalidade concorrencial.

Seguindo a abordagem inicial da formação da estratégia nas organizações, Balestrin e Verschoore (2008), abordam três perspectivas dominantes frente à aplicação e surgimento do conceito da estratégia coletiva: (i) Perspectiva da Estrutura da Indústria, baseada no modelo de Porter (1986), com foco exclusivo na competição; (ii) Visão Baseada em Recursos, destaca que a fonte de vantagem competitiva para uma empresa são seus recursos escassos, que possuem valor, imitabilidade imperfeita e dificuldade de substituição (BARNEY, 1991); (iii) Perspectiva de Custos de Transação, tendo como principal teórico Williamson (1979), constituem questões centrais para a existência desta teoria a falta de confiança nas relações da empresa com seu ambiente e condutas oportunistas de alguns agentes. Neste enfoque, conforme abordado por Balestrin e Verschoore (2008), a estratégia coletiva entra para reduzir o risco e consequentemente os custos de transação.

Bortolaso, Verschoore e Antunes (2012), enfatizam que a construção da estratégia de forma coletiva é crucial para uma rede, visando determinar as políticas de gestão, uso de recursos e posicionamento da rede, na busca de atingir os objetivos propostos na formação da mesma. Neste sentido, é possível obter ganhos superiores quando uma empresa coopera do que quando não coopera com as demais (AXELROD, 1990).

De acordo com Bortolaso, Verschoore e Antunes (2012) a partir da década de 1980 as abordagens das estratégias de cooperação ganharam destaque por meio dos estudos de Astley (1984) e Astley e Fombrun (1983), partindo da ideia de que, além das estratégias iniciais, abordadas nos custos de transação, de produzir internamente ou comprar no mercado, existe um terceiro nível de estratégias que são suportadas por ações conjuntas. Astley (1984, p. 527) coloca que a politica de negócios deveria ter uma nova perspectiva, a estratégia coletiva, definindo "[...] como a formulação conjunta de políticas e implementação de ação por parte dos membros de coletividades interorganizacionais".

As empresas escolhem estratégias de rede para formar relações e alcançar objetivos que dificilmente alcançariam de forma individual e para serem mais competitivas atuando coletivamente (BARBOSA; SACCOMANO; VIEIRA PORTO, 2007). As principais características, que motivam a formação das estratégias coletivas são apresentadas no Quadro 2.

Quadro 2 - Características para formação da estratégia coletiva

\begin{tabular}{|c|l|}
\hline Autores & \multicolumn{1}{|c|}{$\begin{array}{c}\text { Características da Estratégia } \\
\text { Coletiva/Redes }\end{array}$} \\
\hline \multirow{3}{*}{ Nilsen } & $\begin{array}{l}\text { União: acúmulo de recursos financeiros } \\
\text { e de conhecimento, proporcionando } \\
\text { ganhos tanto na redução de custos como } \\
\text { na troca de tecnologias experiências; } \\
\text { Troca: acumulo de conhecimento } \\
\text { através de trocas de serviços; Escala: } \\
\text { não adotar estratégias para prejudicar o } \\
\text { concorrente, mas para cooperar com } \\
\text { ele; Contingência: antever problemas } \\
\text { futuros em mercados de difícil previsão. }\end{array}$ \\
\hline
\end{tabular}




\begin{tabular}{|c|c|}
\hline $\begin{array}{l}\text { Oliver } \\
(1990)\end{array}$ & $\begin{array}{l}\text { Necessidade: empresas obrigadas por } \\
\text { Lei; Assimetria: uma das partes exerce } \\
\text { poder e controle sobre recursos; } \\
\text { Reciprocidade: objetivos comuns; } \\
\text { Eficiência: maior taxa de entrada de } \\
\text { empresas; Estabilidade: prevenir ou } \\
\text { absorver incertezas; Legitimidade: } \\
\text { estabelece imagem da organização. }\end{array}$ \\
\hline $\begin{array}{l}\text { Human; } \\
\text { Provan } \\
\text { (1997) }\end{array}$ & $\begin{array}{l}\text { Trocas interorganizacionais: compras, } \\
\text { vendas, troca de informações; } \\
\text { Credibilidade organizacional: acesso } \\
\text { a novos fornecedores, clientes, etc.; } \\
\text { Acesso a recursos: acesso a novos } \\
\text { mercados e novas ideias; Performance } \\
\text { financeira : benefícios alcançados pelas } \\
\text { empresas na formação da rede. }\end{array}$ \\
\hline $\begin{array}{c}\text { Ebers; Jarillo } \\
\text { (1998) }\end{array}$ & $\begin{array}{l}\text { Aprendizados mútuos: maior agilidade } \\
\text { no desenvolvimento de produtos; } \\
\text { Coespecialidade: novos nichos de } \\
\text { produto e mercado; Melhor fluxo de } \\
\text { informações: reduzir incertezas na } \\
\text { relação; Economia de escala: ganho em } \\
\text { investimentos conjuntos. }\end{array}$ \\
\hline $\begin{array}{l}\text { Dyer; Singh } \\
\text { (1998) }\end{array}$ & $\begin{array}{l}\text { Investimento nas relações: ativos } \\
\text { específicos; Intercâmbio } \\
\text { conhecimento: aprendizagem conjunta; } \\
\text { Complementariedades: rriação } \\
\text { conjunta de novos produtos, processo e } \\
\text { tecnologia; Menos custo de transação: } \\
\text { governança mais eficaz. }\end{array}$ \\
\hline $\begin{array}{l}\text { Marcon; } \\
\text { Moinet } \\
(2000)\end{array}$ & $\begin{array}{l}\text { Fluidez: flexibilidade e adaptabilidade; } \\
\text { Finalidade: motivo da criação da rede; } \\
\text { Capacidade de realizar economias } \\
\text { relacionais: redução da dispersão de } \\
\text { esforços e ganho de tempo; } \\
\text { Capacidade de aprendizagem } \\
\text { coletiva: onde cada um evolui em } \\
\text { função do outro. }\end{array}$ \\
\hline
\end{tabular}

Fonte: Elaborado pelas autoras (2016).

Com base no Quadro 2 é possível observar que as principais características que motivam a formação de redes, na visão da maioria dos autores são as relações de trocas, redução de custos e acesso a novos mercados.

\section{REDES DE COOPERAÇÃO}

As redes podem ser definidas como um conjunto de nós interconectados, possibilitando sua utilização em diversas áreas do conhecimento (FOMBRUN, 1997; CASTELLS, 1999). A intensidade e a frequência da interação entre estes atores sociais são maiores se forem "nós" de uma rede do que se não pertencerem à mesma rede (CASTELLS, 1999).

Whetten (1981) destaca que as relações entre organizações das redes podem ocorrer de quatro formas: a) pares, quando existe uma relação simples de mútua colaboração entre dois nós; b) conjunto de relações, é a soma das relações entre pares; c) conjunto de ações, quando as relações existem e respeitam um funcionamento para atingir um propósito estabelecido; e d) rede, quando o conjunto das relações anteriores é visto de forma macro.

Neste sentido Das e Teng (2002), estabelecem a ideia de rede como uma constelação de organizações, formado por um conjunto de alianças estratégicas que estabelece uma relação competitiva entre organizações e grupos. Jarillo (1988) define redes de cooperação como uma forma de organização que pode ser utilizada por gestores para posicionar suas empresas em um forte ambiente competitivo.

As redes podem assumir diferentes formatos, porém todos caracterizados pela relação de troca entre um número limitado de organizações que mantem o controle de seus recursos individuais e decidem conjuntamente como serão utilizados (EBERS, 1997).

A partir da análise de literaturas nacionais, várias pesquisas apontam para 
vantagens obtidas pelas organizações que atuam em rede, especialmente pequenas empresas que atuam em redes de cooperação. As vantagens deste modelo de gestão organizacional de aprendizagem coletiva são apontadas, por exemplo, por Balestrin e Vargas (2003) e Balestrin e Verschoore (2008), que destacam o alcance de economias de escala, o aumento do poder de barganha que reduz custos de compra e ganhos de representatividade nas negociações com o governo, a ampliação de mercado, a redução dos custos de transação, a realização de economias de escopo pela diversificação do portfólio de produtos e o processo de inovação.

Balestrin e Verschoore (2008) destacam, ainda, que as redes de cooperação possibilitam o desenvolvimento de estratégias coletivas de inovação e permitem o rápido acesso às novas tecnologias pelos seus canais de informação, que dificilmente teriam competido isoladamente no mercado global. As redes de cooperação instituem uma estrutura que gera condições para transações econômicas entre um grupo de agentes (GULATI; GARGIOULO, 1999). Dessa forma uma rede de organizações é formada visando atingir objetivos em comum das organizações que pertencem a esta estrutura.

Balestrin e Vargas (2003), com base em seus estudos, afirmam que não existe um conceito único para redes de cooperação, de forma que não há unanimidade entre os conceitos, porém deve haver uma complementariedade entre eles e não serem excludentes.

Nesse sentido, as redes de cooperação podem se organizar de diversas formas operacionais e de acordo com vários objetivos.
Da mesma forma, diversos autores analisam as tipologias de rede de diferentes perspectivas, conforme pode ser visualizado no Quadro 3.

Quadro 3 - Tipologias de rede de cooperação

\begin{tabular}{|c|c|}
\hline Autores & $\begin{array}{c}\text { Tipologias de redes } \\
\end{array}$ \\
\hline $\begin{array}{l}\text { Santos et. } \\
\text { al. (1994); } \\
\text { Mesquista; } \\
\text { Lazzarini } \\
\text { (2008) }\end{array}$ & $\begin{array}{l}\text { Redes Verticais: composta por empresas } \\
\text { com diferentes funções dentro da cadeia } \\
\text { produtiva. Redes Horizontais: composta } \\
\text { por empresas que trabalham no mesmo } \\
\text { setor ou similares, a cooperação ocorre } \\
\text { entre empresas concorrentes. }\end{array}$ \\
\hline $\begin{array}{c}\text { Grandori; } \\
\text { Soda } \\
(1995)\end{array}$ & $\begin{array}{l}\text { Redes Sociais: não possui contrato } \\
\text { formal; Redes Burocráticas: possui } \\
\text { contrato formal; Redes Proprietárias: } \\
\text { formalização de acordos entre acionistas } \\
\text { e empresas. }\end{array}$ \\
\hline $\begin{array}{c}\text { Belussi; } \\
\text { Arcangeli } \\
\text { (1998) }\end{array}$ & $\begin{array}{l}\text { Redes em estado estacionário: baixo } \\
\text { grau de flexibilidade operacional e } \\
\text { aprendizagem incrementais estáticas; } \\
\text { Redes retráteis e reversíveis: adaptam- } \\
\text { se a mudanças de demanda ou } \\
\text { desenvolvimento tecnológico; Redes } \\
\text { evolutivas: alta flexibilidade, capacidade } \\
\text { criativa e capacidade tecnológica } \\
\text { crescente. }\end{array}$ \\
\hline $\begin{array}{c}\text { Casarotto } \\
\text { Filho } \\
(1998)\end{array}$ & $\begin{array}{l}\text { Foco nas pequenas e médias empresas } \\
\text { (PMEs). Top-Down: empresa menor } \\
\text { fornece sua produção a uma empresa } \\
\text { maior (empresa mãe), exemplo: } \\
\text { terceirização, parcerias e subcontratação; } \\
\text { Redes Flexíveis - consórcios de } \\
\text { pequenas e médias empresas. }\end{array}$ \\
\hline $\begin{array}{c}\text { Marcon; } \\
\text { Moinet } \\
(2000)\end{array}$ & 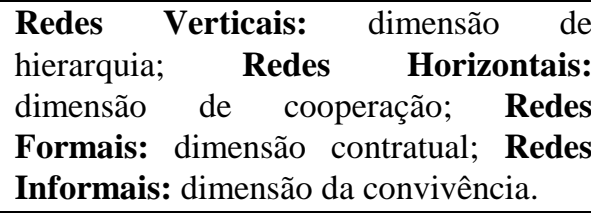 \\
\hline
\end{tabular}

Fonte: Elaborado pelas autoras (2016).

Após a análise conceitual dos temas foco deste estudo, é importante integrar as temáticas comunicação e redes de cooperação, os quais são apresentados na sessão seguinte.

\section{COMUNICAÇÃO EM REDES DE COOPERAÇÃ̃O}

O papel da comunicação organizacional em redes de cooperação é uma ferramenta 
estratégica essencial para as empresas envolvidas, embora a literatura e aplicação empírica que aborde esta temática sejam escassas, com base nas pesquisas efetuadas neste trabalho, destacam-se os estudos de Fernandes (2014) e de Corrêa (2009; 2010). O segundo aborda a temática voltada a disseminar a cultura em redes de organizações, enquanto o primeiro é voltado à comunicação como ferramenta estratégica em redes de cooperação.

A comunicação torna-se um requisito para que ocorra a cooperação, pois é através dela que as organizações interagem. Para Balestrin e Vershoore (2008, p. 151) [...]“"o estabelecimento de redes de cooperação pressupõe três condições fundamentais: objetivos comuns, interação e gestão." Esses elementos são fundamentais para que as empresas obtenham ganhos competitivos. Dentre as condições para formação de redes, abordadas pelos autores, não está especificada a ferramenta de comunicação, porém a mesma pode ser entendida como parte integrante dos requisitos e da gestão da rede (CORREA, 2009).

Através da gestão da comunicação organizacional, visualizada como uma ferramenta de gestão, ocorre a disseminação de objetivos e valores comuns que devem ser partilhados por todos da organização, bem como a divulgação das perspectivas de ganhos competitivos e a interação entre os participantes de uma rede de cooperação (CORREA, 2010). Dessa forma, percebe-se que a relação entre todos os elementos e características de uma rede de cooperação ocorre através da comunicação.

\section{CONSIDERAÇÕES FINAIS}

Este ensaio teórico, que teve como objetivo analisar a relação entre comunicação organizacional, estratégias para formação de redes e redes de cooperação, apresentou uma visão geral acerca dessas definições. A partir das perspectivas teóricas, é possível diagnosticar que os assuntos estão interligados, de forma que a comunicação é um requisito muito importante para a formação de redes de cooperação, sendo parte das ferramentas de gestão. É evidente que existe o sistema informacional tradicional (emissores e receptores) em todas as organizações, entretanto, no caso de redes o estudo da comunicação é um assunto próspero e necessário, uma vez que o processo de comunicação é inerente. Também é importante avaliar de que forma essa comunicação exerce um papel estratégico nas organizações.

Desta forma, as limitações desta pesquisa estiveram centradas na escassez de referencial teórico que aborde a comunicação como ferramenta estratégica em redes de cooperações interorganizacional. Porém, de acordo com os estudos de Correa (2009) e Fernandes (2014), nota-se que existe uma integração entre esses elementos, sendo que ambos os estudos abordam a comunicação como ferramenta de gestão nas redes de cooperação, visto que a comunicação é um requisito para que as organizações cooperem em busca de objetivos comuns, através da integração entre os membros da rede, alcançando vantagem competitiva.

Da mesma forma, sugere-se para estudos futuros a aplicação das abordagens teóricas vistas neste trabalho, em estudos e pesquisas 
empíricas, verificando se realmente a comunicação se constitui em uma ferramenta de estratégia em redes de cooperação.

\section{REFERÊNCIAS}

ASTLEY, W. G. Toward an appreciation of collective strategy. The Academy of Management Review. v.9, n.3, p. 526-535, 1984.

AXELROD, R. The evolution of cooperation. London: Penguin Books, 1990.

BALESTRIN, A.; VARGAS, L. M. A dimensão estratégica das redes horizontais de PMES: teorização e evidências. PPGA/EA/UFRGS, 2003.

BALESTRIN, A.; VERSCHOORE, J. Redes de cooperação empresarial: estratégia de gestão na nova economia. Porto Alegre: Bookman, 2008.

BARBOSA, F. A.; SACOMANO, J. B.; VIEIRA PORTO, A. J. Metodologia para Análise de Redes Interorganizacionais: competitividade e tecnologia. Gestão e Produção. v.14, n. 2, p. 411-423, 2007.

BARNEY, J. B. Firm Resources and Sustainable Competitive Advantage. Journal of Management, v.17, n.1, p. 99-120, 1991.

BELUSSI, F.; ARCANGELI, F. A typology of networks: flexible and evolutionary firms. Research Policy, v. 27, p. 415-428, 1998.

BORTOLASO, I..; VERSCHOORE, J. R.; ANTUNES, J. Estratégias cooperativas: avaliando a gestão da estratégia de redes de pequenas e médias empresas. Revista Brasileira de Gestão e Negócios. v. 14, n.45, p.419-437, 2012.

BUENO, W. Comunicação empresarial e o processo de gestão. 2005. Disponível em <www.comunicacaoempresarial.com.br>. Acesso em 28 Julho 2015.

CASTELLS, M. A sociedade em rede. 8.ed. São Paulo: Paz e Terra, 1999.

CASAROTTO FILHO, N., PIRES, L. H. Redes de pequenas e médias empresas e desenvolvimento local: estratégias para a conquista da competitividade global com base na experiência italiana. São Paulo: Atlas, 1998.

COOK, D. J., MULROW, C.D., RAYNES, R.B. Systematic reviews: synthesis of best evidence for clinical, $1997 . \quad$ Disponível em: <http://www.annals.org/cgi/content/full/126/5/376>. Acesso em: 12 maio 2017.

CORREA, Vera Elisabeth D. A comunicação dirigida como estratégia para disseminar a cultura e a identidade organizacional no contexto das redes de cooperação empresarial. In: Congresso Brasileiro Científico de Comunicação Organizacional e de Relações Públicas - Abrapcorp, 4, 2010, Porto Alegre, RS. Anais (on-line). São Paulo: Abrapcorp, 2010. Disponível: http://www.abrapcorp.org.br/anais2010. Acesso em 20 Jul. 2015.

CORREA, Vera Elisabeth D. Cultura e identidade organizacional disseminadas pela comunicação dirigida: o caso da Redlar. 2009. 119 f. Dissertação (Mestrado em Comunicação e Informação) - UFRGS, Universidade Federal do Rio Grande do Sul, Porto Alegre, 2009.

CRESWELL, J. W. Projeto de pesquisa: métodos qualitativo, quantitativo e misto. 2.ed. São Paulo: Bookman, 2007.

DA SILVA, J. R. G.; OLIVEIRA, M.C.L.

Comunicação Organizacional: oportunidades para uma abordagem de pesquisa interdisciplinar entre a Administração e a área de Estudos da Linguagem. In: Encontro de Estudos Organizacionais da ANPAD, 5, 2008 Belo Horizonte. Anais... Rio de Janeiro: ANPAD, 2008.

DANIELS, Tom D.; SPIKER Barry; PAPA, Michael. Perspectives on organizational communication. Fouth edition. Dubuque, USA: Brown \& Benchmark Publishers, 1997.

DAS, T. K.; TENG, B. Alliance constellations: a social exchange perspective. Academy of Management Review, v.27, n.3, p.445-456, 2002.

DYER, J. H.; SINGH, H. The Relational View: Cooperative Strategy and Sources of Interorganizational Competitive Advantage. Academy Management Review, n.1, v.23, p.660-679, 1998.

EBERS, M.; Explaining interorganizational network formation. In: EBERS, M. (Ed.): The Formation of Interorganizational Networks, Oxford, p. 3-40, 1997.

EBERS, M.; JARILLO, J. C. The construction, forms, and consequences of industry networks. International Studies of Management \& Organization. v. 27, p. 321, Winter 1998.

EISENBERG, Eric M. e GOODALL Jr. H.L. Organizational communication: balancing, creativity and constraint. Second edition. New York, USA: St Martin's Press, 1997. 
FLEURY, A.C.C. FLEURY, M.T.L. Estratégias competitivas e competências essenciais: perspectivas para a internacionalização da indústria no Brasil. Gestão \& Produção. v.10, n.2, p.129-144, 2003.

FERNANDES, E.; I., R. O papel da comunicação estratégica na gestão de redes de cooperação interorganizacionais. Relatório de Estágio (Mestrado em Ciências da Comunicação) - Universidade do Minho, 2014.

FOMBRUN, C. J. Strategies for network research in organizations, Academy of Management Review, v.7, p. 280-291, 1997.

GIL, Antônio C. Como elaborar projetos de pesquisa. 4. ed. São Paulo: Atlas, 2002

GONÇALVES, M. H.S; Comunicações nas organizações: para além da lógica do marketing, a "arte da acção comunicacional". Livro de Actas - IV SOPCOM, p. 503-512, 2005.

GRANDORI, A.; SODA, G.; Inter firm networks: antecedents, mechanism and forms. Organization Studies, v.16, n.2, 1995.

GULATI, R.; GARGIULO, M. Where do interorganizational networks como from? American Journal of Sociology, v.104, n.5, p. 1439-1493, 1999.

HUMAN, S. E.; PROVAN, K. G. An emergent theory of structure and outcomes in small-firm strategic manufacturing network. Academy of Management Journal. v. 40, n. 2, p. 368-403, 1997.

JARILLO, J. C. On strategic networks. Strategic Management Journal. v.9, n.1, p.31-41, 1988.

KREPS, Gary L. Organizational communication: theory and pratice. Second edition. Nova York: Longman, 1990.

KUNSCH, M. M. K. Planejamento de relações públicas na comunicação integrada. São Paulo: Summus, 2006.

MARCON, C.; M.; MOINET, N. La Stratégie-Réseau. Paris: Éditions Zéro Heure, 2000.

MESQUITA, L. F.; LAZZARINI, S. G. Horizontal and vertical relationships in developing economies: implications for SMEs' access to global markets. Academy of Management Journal, v.51, n.2, p.359380, 2008.

NIELSEN, R. P. Cooperative strategy. Strategic Management Journal, v.9, n.5, p.475-492, 1988.
Academy of Management Review, v. 15, n.2, p.241265, 1990.

PEREIRA, Breno A. D.; PEDROZO, Eugenio A. Contribuições à consolidação da teoria estratégica interorganizacional: uma análise dos relacionamentos horizontais. Revista de Administração Contemporânea, v. 9, n. 4, p. 141-161, 2005.

PORTER, Michael E. Estratégia competitiva. Campus: Rio de Janeiro, 1986.

RIEL, Cees. B.M. Principles of corporate communication. Hemel Hempstead, Prenntice - Hall, 1995.

SANTOS, S.A.; PEREIRA, H.J.; ABRAHÃO

FRANÇA, S.E. Cooperação entre as micro e pequenas empresas, SEBRAE/SP, 1994.

SCROFERNEKER, Cleusa Maria Andrade. Perspectivas teóricas da comunicação organizacional. GT Comunicação Organizacional da Intercom, 2000.

WELS, A. M. C.; Estudando a comunicação organizacional: redes e processos integrativos. Conexão Comunicação e Cultura, v.4, n.7, p. 73-86, 2005.

WHETTEN, D. A. Interorganizational relations: a review of the field. Journal of Higher Education, v.52, n.1, p.1-28, 1981.

WILLIAMSON, O. E. Transaction-cost economics: the governance of contractual relations. Journal of Law and Economics, v.22, p.223-261, 1979.

\section{Fernanda Breda}

Mestre em Administração pela Universidade de Passo Fundo - UPF, MBA em Marketing pela Fundação Getúlio Vargas - FGV e Graduação em Jornalismo - UPF.

\section{Elizabete Casagrande Lazarotto}

Mestre em Administração pela Universidade de Passo Fundo - UPF, MBA em Gestão Financeira e Controladoria pela Fundação Meridional - IMED e Graduação em Ciências Contábeis - UPF.

\footnotetext{
Cassiana Maris Lima Cruz

Doutora em Comunicação Social pela PUCRS, Mestre em Administração pela UFSC e professora pesquisadora do Programa de Pós-Graduação em Administração da Universidade de Passo Fundo UPF.
} 


\begin{tabular}{l}
\hline Ana Cláudia Machado Padilha \\
Doutora em Agronegócio (Cepan/UFRGS), Mestre \\
em Agronegócios pela UFRGS e professora \\
pesquisadora na Universidade de Passo Fundo - UPF. \\
\hline \\
Lidiane Zambenedetti \\
Mestre em Administração pela Universidade de \\
Passo Fundo - UPF, Especialização em \\
Contabilidade Pública pela Faculdade Integrada da \\
Grande Fortaleza e Graduação em Ciências \\
Contábeis - UPF.
\end{tabular}

